\title{
LOCALIZED GROUP RINGS, THE INVARIANT BASIS PROPERTY \\ AND EULER CHARACTERISTICS
}

\author{
K. R. GOODEARL ${ }^{1}$
}

\begin{abstract}
The technique of embedding a complex group algebra into the von Neumann regular ring associated with the corresponding $W^{*}$ group algebra is exploited to prove that certain localizations of a group ring $K G$ possess the invariant basis property. From this it follows, using a method of $\mathrm{S}$. Rosset, that certain $K G$-modules have zero Euler characteristic. The assumptions are that $K$ is a commutative integral domain of characteristic zero, and that $G$ has a nontrivial, torsion-free, abelian normal subgroup $A$. The main result of the paper is that the localization of $K G$ obtained by inverting all elements of the form $\alpha-a$, where $\alpha$ is a nonzero element of $K$ and $a$ is a nontrivial element of $A$, has the invariant basis property; more generally, this localization and all its matrix rings are directly finite. (M. Smith has extended the methods of this paper to cover the localization of $K G$ obtained by inverting all nonzero elements of $K A$.) Given a $K G$-module $M$ which has a finite free resolution, such that $M$ is finitely generated over $K$, it is proved that the Euler characteristic of $M$ is zero. This verifies an unpublished result of Rosset.
\end{abstract}

We thank S. Gersten for bringing this problem to our attention.

All rings and algebras considered in this paper are assumed to be associative with unit.

DefinItION. A ring $R$ is said to have the invariant basis property if the rank of every finitely generated free $R$-module is unique. In other words, for all positive integers $m$ and $n$, the free right (or left) $R$-modules $R^{m}$ and $R^{n}$ must not be isomorphic unless $m=n$.

DEFINITION. A ring $R$ is said to be directly finite (or von Neumann finite) provided all one-sided inverses in $R$ are two-sided, i.e., any $x, y \in R$ satisfying $x y=1$ must also satisfy $y x=1$.

The endomorphism ring of a module $M$ is directly finite if and only if $M$ is not isomorphic to any proper direct summand of itself [3, Lemma 6.9]. For a ring $R$ to have the invariant basis property, it certainly suffices to know that no finitely generated free $R$-module is isomorphic to a proper direct summand of itself. Since the endomorphism ring of a free module $R^{n}$ is isomorphic to the $n \times n$ matrix ring $M_{n}(R)$, the invariant basis property for $R$ may be obtained from direct finiteness of

Received by the editors May 23, 1983 and, in revised form, November 4, 1983.

1980 Mathematics Subject Classification. Primary 16A27.

Key words and phrases. Group ring, invariant basis property, direct finiteness, Euler characteristic.

${ }^{1}$ This work was partially supported by a National Science Foundation grant.

(i) 1984 American Mathematical Society $0002-9939 / 84 \$ 1.00+\$ .25$ per page 
the matrix rings over $R$. Namely

LEMMA 1. If $R$ is a ring such that all the matrix rings $M_{n}(R)$ are directly finite, then $R$ has the invariant basis property.

Definition. A right denominator set (or right Ore set) in a ring $R$ is a multiplicatively closed subset $X \subseteq R$ such that:

(i) Given any $x \in X$ and $r \in R$, there exist $y \in X$ and $s \in R$ satisfying $x s=r y$.

(ii) Given any $x \in X$ and $r \in R$ satisfying $x r=0$, there exists $y \in X$ such that $r y=0$.

Definition. Given a right denominator set $X$ in a ring $R$, there exists a right ring of fractions (or right Ore localization) $R\left[X^{-1}\right]$ and a canonical ring homomorphism $\varphi: R \rightarrow R\left[X^{-1}\right]$ such that:

(i) For any $x \in X$, the element $\varphi(x)$ is invertible in $R\left[X^{-1}\right]$.

(ii) $R\left[X^{-1}\right]=\left\{\varphi(r) \varphi(x)^{-1} \mid r \in R\right.$ and $\left.x \in X\right\}$.

(iii) $\operatorname{ker}(\varphi)=\{r \in R \mid r x=0$ for some $x \in X\}$.

(See [14, Proposition II.1.4].) If the elements of $X$ are all non-zero-divisors in $R$, the kernel of $\varphi$ is zero. In this case, we identify $R$ with the subring $\varphi(R)$ in $R\left[X^{-1}\right]$.

The left ring of fractions $\left[X^{-1}\right] R$, for a left denominator set $X$ in a ring $R$, is defined symmetrically. If $X$ is both a right and a left denominator set, $R\left[X^{-1}\right]$ and $\left[X^{-1}\right] R$ may be identified [14, Corollary II.1.3].

Definition. Let $K$ be a ring and $A$ a nontrivial group. We define $X(K, A)$ to be the subset of the group ring $K A$ consisting of all products

$$
\left(\alpha_{1}-a_{1}\right)\left(\alpha_{2}-a_{2}\right) \cdots\left(\alpha_{n}-a_{n}\right),
$$

where the $\alpha_{i}$ are non-zero-divisors in $K$ and the $a_{i}$ are nontrivial elements of $A$.

Lemma 2. Let $K$ be a commutative ring, let $G$ be a group, and let $A$ be a nontrivial, torsion-free, abelian normal subgroup of $G$. Then $X(K, A)$ is a right and left denominator set of non-zero-divisors in the group ring $K G$.

Proof. Consider a non-zero-divisor $\alpha \in K$, a nontrivial element $a \in A$, and an element $r \in K G$ such that $(\alpha-a) r=0$. If $r(g)$ denotes the coefficient of a group element $g$ in $r$, then $r\left(a^{-1} g\right)=\alpha r(g)$ for any $g \in G$. Hence, $r\left(a^{-n} g\right)=\alpha^{n} r(g)$ for all integers $n$. Since $r$ has finite support while $a$ has infinite order, $r(g)=0$. Thus $r=0$. Similarly, $r(\alpha-a)=0$ implies $r=0$, proving that $\alpha-a$ is a non-zero-divisor in $K G$.

Therefore $X(K, A)$ is a multiplicatively closed set of non-zero-divisors in $K G$.

Now let $x \in X(K, A)$ and $r \in K G$. Write $r=\alpha_{1} g_{1}+\cdots+\alpha_{n} g_{n}$ for some $\alpha_{i} \in K$ and some $g_{i} \in G$. Each of the elements $g_{i}^{-1} x g_{i}$ lies in $X(K, A)$ because $A$ is normal in $G$, and so the element

$$
y=\left(g_{1}^{-1} x g_{1}\right)\left(g_{2}^{-1} x g_{2}\right) \cdots\left(g_{n}^{-1} x g_{n}\right)
$$

lies in $X(K, A)$. Since $K A$ is commutative, the elements $g_{i}^{-1} x g_{i}$ commute with each other. Hence, $y=g_{i}^{-1} x g_{i} z_{i}$ for each $i$, where $z_{i} \in X(K, A)$. Then

$$
x\left(\sum \alpha_{i} g_{i} z_{i}\right)=\Sigma \alpha_{i} g_{i} g_{i}^{-1} x g_{i} z_{i}=\Sigma \alpha_{i} g_{i} y=r y .
$$


Therefore $X(K, A)$ is a right denominator set in $K G$. (The second condition is trivially satisfied because $X(K, A)$ consists of non-zero-divisors.) By symmetry, $X(K, A)$ is also a left denominator set.

The right ring of fractions $(K G)\left[X(K, A)^{-1}\right]$ may now be formed (keeping the notation of Lemma 2). Provided $K$ is an integral domain of characteristic zero, we shall prove that this ring and all its matrix rings are directly finite. Our proof is an extension of the method by which Kaplansky first proved that $K G$ is directly finite [5, p. 122]. Modulo an easy reduction, only the case in which $K=\mathbf{C}$ needs to be considered. Following Kaplansky's idea, we embed $\mathbf{C} G$ in a $W^{*}$-algebra, i.e., a weakly closed selfadjoint algebra of bounded linear operators on a complex Hilbert space, as follows (see $[\mathbf{1 1}, \mathbf{1 5}]$ for general information about $W^{*}$-algebras).

First, topologize $G$ using the discrete topology. Next, form the Hilbert space $L^{2}(G)$ of all square-summable complex-valued functions on $G$. The set $G$ may be embedded in $L^{2}(G)$ by identifying each element $g \in G$ with the characteristic function of the singleton $\{g\}$. Then $G$ is an orthonormal basis for $L^{2}(G)$. Left multiplication by any element $g \in G$ induces a unitary transformation $T_{g}$ on $L^{2}(G)$. If we identify each $g \in G$ with the corresponding operator $T_{g}$, then $G$ becomes identified with a subgroup of the unitary group of the algebra $B\left(L^{2}(G)\right)$ of all bounded linear transformations on $L^{2}(G)$. The elements of $G$ are linearly independent in $B\left(L^{2}(G)\right)$, and hence we may identify $C G$ with the subalgebra of $B\left(L^{2}(G)\right)$ generated by $G$. As $g^{*}=g^{-1}$ for all $g \in G$, we see that $C G$ is a selfadjoint subalgebra of $B\left(L^{2}(G)\right)$. Finally, let $W(G)$ be the weak closure of $C G$ in $B\left(L^{2}(G)\right)$. Then $W(G)$ is a $W^{*}$-algebra, which we may call the $W^{*}$ group algebra of $G$. The embedding of $G$ into $W(G)$ is the left regular unitary representation of $G$ (see also [2, pp. 191,192 or 8, p. 13]).

DEFINITION. Let $p$ and $q$ be projections (i.e., selfadjoint idempotents) in a $W^{*}$-algebra $W$. If there exists an element $v \in W$ such that $v v^{*}=p$ and $v^{*} v=q$, then $p$ and $q$ are said to be ${ }^{*}$-equivalent. The algebra $W$ is of finite type (or just "finite") if 1 is the only projection in $W$ that is *-equivalent to 1 . Equivalently, $W$ is of finite type if and only if every $x \in W$ satisfying $x x^{*}=1$ also satisfies $x^{*} x=1$. It can be proved that every $W^{*}$-algebra of finite type is directly finite (a well-known fact without adequate references), but we shall not require this result.

THEOREM 3. If $G$ is any discrete group, $W(G)$ is of finite type.

Proof [8, Theorem 2.4]. The countable case may also be found in [2, Proposition 16, p. 192; 7, Theorem, p. 61].

Lemma 4. Let $G$ be a discrete group, let $u \in G$, and let $\alpha \in \mathbf{C}$. If $u$ has infinite order, then $\alpha-u$ is a non-zero-divisor in $W(G)$.

Proof. We may obviously assume that $\alpha \neq 0$. Given any $f \in L^{2}(G)$ for which $(\alpha-u)(f)=0$, we observe that $f\left(u^{-1} g\right)=\alpha f(g)$ for any $g \in G$. Hence, $f\left(u^{-n} g\right)=$ $\alpha^{n} f(g)$ for all integers $n$. Since $u$ has infinite order,

$$
\sum_{n \in \mathbf{Z}}|\boldsymbol{\alpha}|^{2 n}|f(g)|^{2}=\sum_{n \in \mathbf{Z}}\left|f\left(u^{-n} g\right)\right|^{2} \leqslant \sum_{h \in G}|f(h)|^{2}<\infty,
$$


whence $f(g)=0$. Thus $f=0$, which proves that $\alpha-u$ is injective. The operator $(\alpha-u)^{*}=\bar{\alpha}-u^{-1}$ is similarly injective.

If $w \in W(G)$ with $(\alpha-u) w=0$, then $w=0$ because $\alpha-u$ is injective. If $w(\alpha-u)=0$, then $(\alpha-u)^{*} w^{*}=0$ and so $w^{*}=0$, whence $w=0$.

Murray and von Neumann proved in [9] that any $W^{*}$-algebra $W$ of finite type can be embedded in a (von Neumann) regular ring $U(W)$ whose elements are closed, densely defined, unbounded operators affiliated with $W$ in a certain sense. For an exposition of the construction of $U(W)$ using modern terminology, see [1, pp. 150, 151]. We shall need the facts that $U(W)$ is a complex *-algebra containing $W$ as a *-subalgebra, that $U(W)$ is a *-regular ring (i.e., every principal right or left ideal of $U(W)$ can be generated by a projection), and that all projections in $U(W)$ actually lie in $W$. (The first statement is in [1, Theorem 1], and the second is shown at the beginning of $[1, \S 3]$. If $p$ is any projection in $U(W)$, then $p^{*} p+(1-p)^{*}(1-p)=1$, and hence $p \in W$ by [1, Theorem 1].)

Lemma 5. Let $G$ be a discrete group, let $u \in G$, and let $\alpha \in \mathbf{C}$. If $u$ has infinite order, then $\alpha-u$ is invertible in $U(W(G))$.

Proof. Set $x=\alpha-u$ and $Q=U(W(G))$. As $Q$ is *-regular, there exist projections $p$ and $q$ in $Q$ such that $x Q=p Q$ and $Q x=Q q$. Then $(1-p) x=0$ and $x(1-q)=0$. As $1-p$ and $1-q$ lie in $W(G)$, and $x$ is a non-zero-divisor in $W(G)$ (Lemma 4), we obtain $1-p=1-q=0$. Therefore $x Q=Q x=Q$, whence $x$ is invertible in $Q$.

Readers familiar with maximal quotient rings may prefer to prove Lemma 5 using the facts that $W(G)$ is a right and left nonsingular ring whose maximal right and left quotient rings coincide with $U(W(G))$ [1, Lemma 3 and Theorem 3]. It follows that every non-zero-divisor in $W(G)$ is also a non-zero-divisor in $U(W(G))$, and hence (using Lemma 4) that $\alpha-u$ is a non-zero-divisor in $U(W(G))$. As $U(W(G))$ is a regular ring, $\alpha-u$ is thus invertible in $U(W(G))$.

Our main result is proved by working within $U(W(G))$, as follows.

THEOREM 6. Let $K$ be a commutative integral domain of characteristic zero, let $G$ be a group, and let $A$ be a nontrivial, torsion-free, abelian normal subgroup of $G$. If $R=(K G)\left[X(K, A)^{-1}\right]$, then all the matrix rings $M_{n}(R)$ are directly finite, and $R$ enjoys the invariant basis property.

Proof. By Lemma 1, it suffices to prove the direct finiteness. As any two matrices over $R$ are also matrices over $(F G)\left[X(F, A)^{-1}\right]$ for some countable subring $F$ of $K$, the problem reduces to the case where $K$ is countable. In this case, the quotient field of $K$ is isomorphic to a subfield of $\mathbf{C}$, and hence $R$ is isomorphic to a subring of $(\mathbf{C} G)\left[X(\mathbf{C}, A)^{-1}\right]$. Thus we may assume, without loss of generality, that $K=\mathbf{C}$.

If $Q=U(W(G))$, then by Lemma 5 all elements of $X(K, A)$ are invertible in $Q$. Consequently, the inclusion map $K G \rightarrow Q$ extends to a ring homomorphism $\varphi$ : $R \rightarrow Q$ [14, Proposition II.1.1], and it is clear that $\varphi$ is an embedding. Hence, it suffices to show that all the matrix rings $M_{n}(Q)$ are directly finite. 
By [1, Lemma 1], the regular ring $Q$ is unit-regular (i.e., for each $x \in Q$, there exists an invertible element $u \in Q$ such that $x u x=x$ ). Therefore $M_{n}(Q)$ is directly finite, by [4, Proposition 5.2].

M. K. Smith has extended the conclusions of Theorem 6 to a somewhat larger localization of $K G$. Namely, if $Y$ is the set of nonzero elements of $K A$, then $Y$ is a right and left denominator set of non-zero-divisors in $K G$ (as in [10, Lemma 13.3.5(ii)]), and all the matrix rings over $(K G)\left[Y^{-1}\right]$ are directly finite [12, Corollary 3]. The difficult part is to prove that all the elements of $Y$ are non-zero-divisors in $W(G)$ (cf. Lemma 4).

As an application of Theorem 6, we show how it supports a method devised by S. Rosset to investigate Euler characteristics of $K G$-modules.

Definition. Let $M$ be a module over a ring $R$. A finite free resolution of $M$ is any exact sequence

$$
0 \rightarrow F_{n} \rightarrow F_{n-1} \rightarrow \cdots \rightarrow F_{1} \rightarrow M \rightarrow 0
$$

where each $F_{i}$ is a finitely generated free $R$-module. If $M$ has a finite free resolution, and if $R$ has the invariant basis property, the Euler characteristic of $M$ is defined to be the number

$$
\sum_{i=1}^{n}(-1)^{i+1} \operatorname{rank}\left(F_{i}\right) \text {. }
$$

The Euler characteristic of $M$ is independent of the choice of the finite free resolution [6, pp. 138, 139].

Since any group ring over a commutative ring has a homomorphic image which is a field, such a group ring has the invariant basis property. Thus the Euler characteristic is defined for any module with a finite free resolution over any such group ring.

THEOREM 7. Let $K$ be a commutative integral domain of characteristic zero, let $G$ be a group and let $M$ be any right KG-module which has a finite free resolution. Assume that $M$ is finitely generated as a $K$-module. If $G$ has a nontrivial, torsion-free, abelian normal subgroup $A$, then the Euler characteristic of $M$ is zero.

Proof. The existence of a finite free resolution for $M$ and the value of the Euler characteristic of $M$ remain unchanged after tensoring everything with the algebraic closure of the quotient field of $K$. Thus there is no loss of generality in assuming that $K$ is an algebraically closed field.

Now set $R=(K G)\left[X(K, A)^{-1}\right]$. As a left $K G$-module, $R$ is the directed union of the submodules $(K G) x^{-1}$ (for $x \in X(K, A)$ ), each of which is a free left $K G$-module (of rank 1). Hence, $R$ is a flat left $K G$-module, and so any finite free resolution for $M$ tensors up to a finite free resolution for $M \otimes_{K G} R$. As $R$ has the invariant basis property (Theorem 6), the Euler characteristic of $M \otimes_{K G} R$ is defined. Note that $M \otimes_{K G} R$ has the same Euler characteristic as $M$.

Choose a nontrivial element $a \in A$. As $M$ is finite dimensional over $K$, there exists a nonconstant polynomial $p(t)$ over $K$ such that $M p(a)=0$. Since $K$ is algebraically closed, $p(t)$ is a product of linear factors, and hence $p(a)=\alpha a^{n} x$ for some nonzero 
$\alpha \in K$, some nonnegative integer $n$, and some $x \in X(K, A)$. Consequently, $p(a)$ is invertible in $R$, whence $M \otimes_{K G} R=0$.

Thus the Euler characteristic of $M \otimes_{K G} R$ is zero, and therefore the Euler characteristic of $M$ is zero.

One case of Theorem 7 is contained in a result of Stallings [13, Corollary 3.6], namely, the case in which $M=K$ (with the trivial $G$-action) and $A$ is the center of $G$. The general result is contained in unpublished work of Rosset.

An alternate proof of Theorem 7 may be obtained by replacing $X(K, A)$ with the set of nonzero elements of $K A$. Then $K$ need not be replaced by an algebraically closed field, and $p(a)$ need not be factored. However, [12, Corollary 3] must be used instead of Theorem 6 . We have used $X(K, A)$ in order to reduce the total amount of work needed to reach Theorem 7 .

NOTE ADDED IN PROOF. Rosset's results have now appeared in $A$ vanishing theorem for Euler characteristics, Math. Z. 185 (1984), 211-215.

\section{REFERENCES}

1. S. K. Berberian, The maximal ring of quotients of a finite von Neumann algebra, Rocky Mountain J. Math. 12 (1982), 149-164.

2. S. A. Gaal, Linear analysis and representation theory, Grundlehren der Math. Wissenschaften, Band 198, Springer-Verlag, Berlin, Heidelberg and New York, 1973.

3. K. R. Goodearl, Ring theory: nonsingular rings and modules, Dekker, New York, 1976.

4. __ Von Neumann regular rings, Pitman, London, 1979.

5. I. Kaplansky, Fields and rings, Univ. of Chicago Press, Chicago, 1969.

6. Commutative rings, Allyn \& Bacon, Boston, Mass., 1970.

7. G. W. Mackey, The theory of unitary group representations, Univ. of Chicago Press, Chicago, 1976.

8. F. L. Mautner, Unitary representations of locally compact groups. I, Ann. of Math. (2) 51 (1950), $1-25$.

9. F. J. Murray and J. von Neumann, On rings of operators, Ann. of Math (2) 37 (1936), 116-229.

10. D. S. Passman, The algebraic structure of group rings, Interscience, New York, 1977.

11. J. T. Schwartz, $W^{*}$-algebras, Gordon \& Breach, New York, 1967.

12. M. K. Smith, Central zero-divisors in group algebras, Proc. Amer. Math. Soc. 91 (1984), 529-531.

13. J. Stallings, Centerless groups - an algebraic formulation of Gottlieb's theorem, Topology 4 (1965), $129-134$.

14. B. Stenström, Rings of quotients, Grundlehren der Math. Wissenschaften, Band 217, Springer-Verlag, Berlin, Heidelberg and New York, 1975.

15. D. M. Topping, Lectures on Von Neumann algebras, Van Nostrand Reinhold, New York, 1971.

Department of Mathematics, University of Utah, Salt lake City, Utah 84112 